\title{
VIDEOLAPAROSCOPIA NO TRATAMENTO DA DOENÇA POR REFLUXO GASTROESOFÁGICO
}

\section{VIDEOLAPAROSCOPY IN THE TREATMENT OF ESOPHAGEAL REFLUX DISEASE}

\author{
Hamilton Luiz Xavier Funes, TCBC-SP ${ }^{1}$ \\ Gilberto Kenichi Anai ${ }^{1}$ \\ Manoel Carlos Libano dos Santos, ACBC-SP ${ }^{1}$ \\ Antonio Pelosi de Moura Leite, TCBC-SP2 \\ Fabiano Corrêa Salvador ${ }^{3}$
}

\begin{abstract}
RESUMO: A doença por refluxo gastroesofágico (DRGE) é uma entidade relativamente recente que vem se tornando freqüente na prática clínica, haja vista que responde por $75 \%$ das doenças de esôfago. A introdução rotineira de métodos investigatórios mais apurados, como a pHmetria de 24 horas e a esofagomanometria, aliadas à endoscopia com biópsia, trouxeram ao cirurgião dados que lhe permitiram utilizar com segurança a videolaparoscopia na cura cirúrgica desta afecção. A confecção de válvulas anti-refluxo, parciais ou totais, proporcionam resultados bastante animadores. Na série apresentada, foram estudados 30 pacientes operados por videolaparoscopia, num período de 36 meses, com excelentes resultados, sendo que apenas um paciente, após seis meses de cirurgia, apresentou, à endoscopia de controle, hérnia de hiato, com esofagite leve, mas absolutamente assintomática. Concluindo, os resultados mostraram que a fundoplicatura por via laparoscópica é um método seguro para a cura da doença por refluxo gastroesofágico, estando indicada em suas complicações e em pacientes refratários ao tratamento clínico, pelo baixo índice de morbimortalidade apresentado.
\end{abstract}

Descritores: Doença por refluxo gastroesofágico; Esfíncter esofágico inferior; Refluxo gastroesofágico; Esofagite por refluxo; Videolaparoscopia.

\section{INTRODUÇÃO}

A doença por refluxo gastroesofágico (DRGE) é uma entidade freqüente na prática clínica e responde por $75 \%$ das doenças do esôfago, sendo a condição mais freqüente nos Estados Unidos ${ }^{1}$. Pelo menos 10 bilhões de dólares são gastos anualmente na prescrição de drogas para queimação e dispepsia ${ }^{2}$. Como sabemos, a ocorrência de refluxo de material gástrico para esôfago não significa necessariamente a presença de inflamação da mucosa (esofagite), já que o refluxo pode ocorrer em condições fisiológicas, principalmente associado à deglutição. A utilização de métodos investigatórios mais apurados, tais como a pHmetria e esofagomanometria, vieram trazer um outro alento ao diagnóstico preciso desta afecção.
Após o sucesso da aplicação da laparoscopia na doença biliar, outros procedimentos puderam ser realizados pelo método laparoscópico; assim, em 1991, Dallèmagne realizou a primeira fundoplicatura laparoscópica e o sucesso dessa cirurgia em seguimentos precoces tem sido rotulado por vários autores como eficaz ${ }^{3}$.

O objetivo deste estudo é mostrar que, com essa nova via de acesso e técnica, o trauma sofrido pelo paciente é mínimo, levando a uma alta hospitalar precoce e a um rápido retorno do paciente às suas atividades profissionais, aliados a um índice muito baixo de morbimortalidade.

\section{MÉTODO}

Durante o período de abril de 1996 a abril de 1999 , foram submetidos ao método 30 pacientes, sendo 16 (53\%)

1. Cirurgião do Instituto de Gastroenterologia Rio Preto-SP.

2. Cirurgião convidado.

3. Residente do Instituto de Gastroenterologia Rio Preto-SP.

Recebido em 31/5/99

Aceito para publicação em 2/5/2000

Trabalho realizado no Instituto de Gastroenterologia Rio Preto-SP. 
do sexo masculino e 14 (46\%) do sexo feminino. A idade variou de três a 70 anos, com média global de idade de 44.1 anos.

Todos os pacientes apresentavam o diagnóstico de doença por refluxo gastroesofágico após longo, exaustivo e dispendioso tratamento clínico sem sucesso, cujas principais manifestações clínicas eram pirose e regurgitação.

Apenas um paciente apresentava colelitíase associada, tratada no mesmo ato cirúrgico. Os pacientes foram encaminhados para consulta ambulatorial, mantidos em rigorosa observação clínica e submetidos a pHmetria de 24 horas (20 pacientes); endoscopia com biópsia (25 pacientes), com grau de esofagite, conforme Savary-Miller; esofagomanometria (oito pacientes) e tomografia computadorizada em um paciente. Não foram realizadas medidas do $\mathrm{pH}$ esofágico nos dois primeiros pacientes, por não possuirmos, à época, equipamento adequado e por ter havido impossibilidade técnica em duas crianças de três e oito anos, que não aceitaram o exame.

O ato cirúrgico foi realizado sob anestesia geral, com monitorização total dos pacientes, principalmente oxicarbinometria. Os pacientes ficaram em posição de litotomia, com o cirurgião à sua frente, sendo usados cinco trocartes, obedecendo à seguinte disposição :

O primeiro, de $10 \mathrm{~mm}$, supra-umbilical, para instalação de óptica de $30^{\circ}$; o segundo, de $5 \mathrm{~mm}$, subxifóideo, para afastar o fígado; o terceiro, de $10 \mathrm{~mm}$, no hipocôndrio esquerdo; o quarto, de $5 \mathrm{~mm}$, no hipocôndrio direito, e o quinto, de $5 \mathrm{~mm}$, no flanco esquerdo (Figura 1).

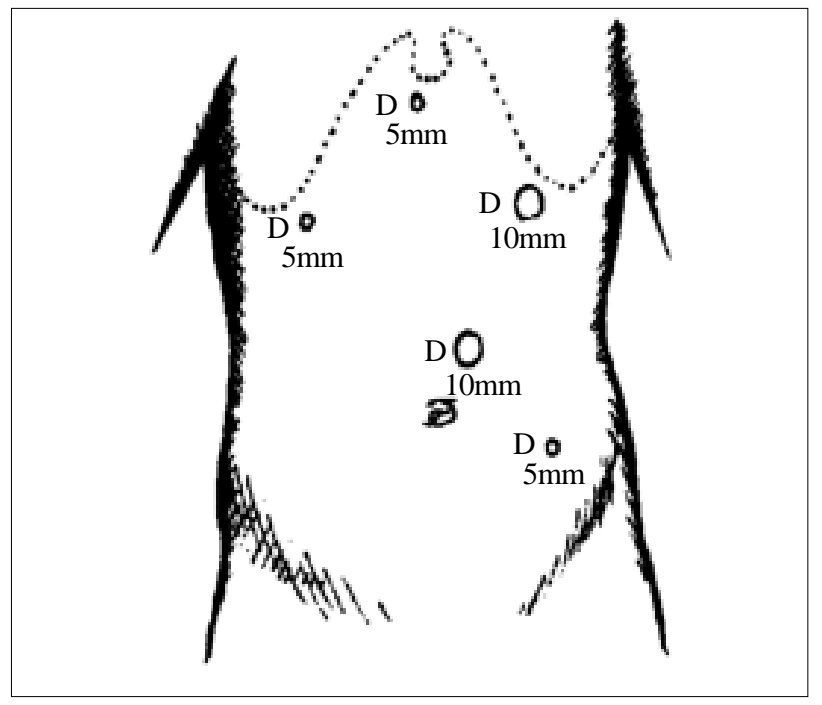

Figura 1 - Representação esquemática das punções

O peritônio que recobre a transição esofagogástrica era seccionado, tomando-se o cuidado de preservar o ramo hepático do nervo Vago. Fazia-se a dissecção dos braços esquerdo e direito do pilar diafragmático e do nervo Vago posterior. Colocava-se um dreno de Penrose circundando o esôfago, com a finalidade de facilitar a mobilização da sua porção terminal. Ligavam-se os vasos curtos do estômago, que, a nosso ver, facilitava sobremaneira a confecção da válvula. Fazia-se hiatoplastia com dois a três pontos, envolvendo em " $\mathrm{X}$ " os dois braços do pilar diafragmático, com fio inabsorvível.

Para a feitura da válvula, era inicialmente confeccionado um ponto, abrangendo as duas extremidades do estômago, que passavam posteriormente ao esôfago, sem que o esôfago fosse envolvido na sutura. A seguir, mais dois a três pontos abaixo do ponto inicial, desta vez abrangendo, além das duas extremidades do estômago, a parede esofágica, completando a válvula. Todos os pacientes saíram da sala de cirurgia sem sonda nasogástrica, mas de maneira rotineira, utilizando antieméticos do tipo ondansetron no pós-operatório imediato. A ingesta oral líquida era liberada no primeiro dia pós-operatório, a alta hospitalar no segundo dia de pós-operatório. Recomendamos, durante os primeiros 30 dias de pós-operatório, cisaprida e dieta semilíquida, cuja liberação se dará após endoscopia de controle, no $30^{\circ}$ dia pós operatório.

\section{RESULTADOS}

O tempo médio de cirurgia ficou situado em 140 minutos, não tendo sido registrado nenhum acidente operatório e, durante a hospitalização, não ocorreu dor ou vômito. Não houve conversão para o método aberto, nem mortalidade. Não observamos casos de infecção da ferida operatória e os pacientes receberam alta hospitalar sem queixa após 48 horas de pós-operatório.

Todos os pacientes tiveram seu seguimento ambulatorial feito pelo cirurgião que os operou; o mais antigo com 35 meses de operado e o mais recente com 10 meses. A anamnese foi dirigida para os sintomas anteriormente relatados pelo paciente. Apenas dois pacientes apresentaram algo digno de nota: um deles, no sexto mês pós-operatório, na endoscopia de controle, mostrou hérnia hiatal e esofagite leve, mas assintomática. $\mathrm{O}$ outro, após 30 dias de cirurgia, mediante transgressão alimentar e alcoólica, impactou fragmento de $10 \mathrm{~cm}$ de carne no esôfago médio, sendo prontamente retirado por via endoscópica.

Os demais pacientes se apresentam totalmente livres de sintomas e inteiramente reintregrados às suas funções cotidianas.

\section{DISCUSSÃO}

Atualmente, DRGE é o termo preferido, onde se agrupam todos os sintomas conseqüentes ao refluxo gastroesofágico, que por sua vez é reconhecido como tendo grande diversidade de apresentações clínicas ${ }^{4}$.

Queimação retroesternal e regurgitação foram os sintomas mostrados com especificidade de $92 \%$ como indicadores da doença de refluxo ${ }^{4}$. Em nosso material, tivemos a confirmação exata dos dados da literatura, com a queimação retroesternal se tornando o sintoma predominante. Outros sintomas, além da queimação retro esternal, podem ocorrer; tais como dor torácica, asma, tosse crônica, laringite e rouquidão ${ }^{1}$. Cerca de $30 \%$ dos portadores de dor pré-cordial, 
tipo angina, não apresentam alterações cardiológicas. Metade desses pacientes apresentam refluxo importante ${ }^{5}$.

Nosso paciente mais idoso apresentava, de forma suave, sintomas pertinentes ao refluxo gastroesofágico, mas preponderantemente tosse, bronco espasmo e episódios freqüentes de pneumonia. Fato idêntico foi observado em nosso paciente mais jovem, que, desde o nascimento, apresentava refluxo gastroesofágico, acompanhado de episódios repetidos de pneumonia durante o primeiro ano de vida.

No que diz respeito à correlação clínico-endoscópica, é sabido que $40 \%$ a $60 \%$ dos pacientes portadores de DRGE têm exame endoscópico considerado normal ${ }^{6}$. Tal fato pode ser explicado pela diferença existente entre os critérios europeu e norte-americano na conceituação dos achados endoscópicos, que devem ser valorizados no diagnóstico das esofagites, especialmente de graus menores $^{7}$. Biópsias realizadas em pacientes com refluxo e endoscopia normal aumentam em $10 \%$ a $15 \%$ a sensibilidade diagnóstica ${ }^{8}$.

Em nosso material, foram realizadas 25 endoscopias, acompanhadas de biópsia em 16 pacientes. As biópsias revelaram predominância de esofagite crônica, seguida de neoplasia escamosa epidermóide e esofagite erosiva.

No que diz respeito à esofagomanometria como meio auxiliar no diagnóstico da DRGE, podemos dizer que o esfíncter esofagiano inferior (E.E.I) é importante na prevenção do refluxo. Uma hipotonia do mesmo se traduz por uma pressão esfincteriana diminuída, menor ou igual a $25 \mathrm{mmHg}$ e é uma causa inevitável de refluxo ${ }^{9}$. Pacientes que apresentam E.E.I incompetente, normalmente respondem mal à terapia com drogas ${ }^{10} \mathrm{e}$ apresentam refluxo patológico com sintomatologia ${ }^{11}$. Nossos pacientes, apresentaram, na esofagomanometria, hipotonia do E.E.I, diminuição da amplitude do corpo em um paciente e ondas secundárias em um paciente.

Os objetivos principais da esofagomanometria préoperatória são: comprovar a doença, constatar a existência de dificuldades no manejo clínico, relacionar doença com sintomas (típicos ou atípicos) e assegurar-se das condições funcionais do esôfago em se adaptar à uma válvula ${ }^{12}$.

Quanto à pHmetria esofágica de 24 horas, o escore de Demeester \& Johnson fornece sensibilidade de 90,3\% e especificidade de $90,0 \%$ para o diagnóstico da DRGE ${ }^{12}$, mas, infelizmente, a pHmetria isolada não se presta para o diagnóstico de esofagite de refluxo e, por isso, não substitui o estudo endoscópico, mas Jamieson et al. ${ }^{13}$, por sua vez, citam a pHmetria prolongada de 24 horas como o exame mais sensível e específico para o diagnóstico da DRGE.

Na fisiopatologia da DRGE, a duração do refluxo é mais importante do que o seu número. Em nosso material, todos os 20 pacientes que se submeteram à pHmetria de 24 horas apresentavam refluxos com duração superior a seis minutos, sendo que, em um deles (o mais idoso, com episódios repetidos de pneumonia), foi constatado um refluxo com duração superior a 50 minutos. No nosso estudo, o pH esofágico de 24 horas foi realizado em 20 pacientes, predominando o refluxo patológico em 15 e fisiológico em cinco pacientes. Nossos pacientes apresentaram média de 54 refluxos com $\mathrm{pH}<4$.

A escolha do tipo de válvula a ser realizada é um item de suma importância e equivale à escolha do paciente para o tratamento cirúrgico. A fundoplicatura à Nissen em nosso material tem sido a preferida, apresentando todas as vantagens de um procedimento minimamente invasivo ${ }^{14}$. O floppy Nissen, em nosso estudo, tem se mostrado uma operação efetiva, com baixa incidência de efeitos colaterais e sem tendência a falhas tardias ${ }^{15}$. Uma válvula de 2 a $3 \mathrm{~cm}$, frouxa, é suficiente para evitar o refluxo.

Finalizando, podemos afirmar que o procedimento laparoscópico surgiu para manter os pacientes livres dos sintomas da DRGE refratários ao tratamento clínico, com rápida recuperação e baixas taxas de morbidade, com mortalidade de $0,5 \% 10,14$.

$\mathrm{Na}$ criança, reservamos o tratamento cirúrgico apenas para os casos de doença grave, sem resposta ao tratamento clínico.

\begin{abstract}
The aim of this study is to show the effectiveness of videolaparoscopic fundoplication as the definitive treatment of GERD. From April 1996 to April 1999, 30 patients underwent fundoplication and hiatoplasty for the treatment of reflux esophagitis through videolaparoscopy. Sixteen (53\%) were males and 14 (46\%) females. The mean age was 44.1, ranging from 3 to 70 years old. Indications for surgery were refractory GERD after prolonged clinical treatment and paraesophageal hernia with hemorrhage $(0.3 \%)$. Five trocars were employed for each surgical procedure. Hiatoplasty was performed with 00 cotton sutures, wrapping with a X both arms of the diaphragmatic pillars. For fundoplication, after the tranposition of the gastric fundus behind the abdominal portion of the esophagus, an inicial suture was set on the two gastric extremities of the anterior wall, without passing through the esophagus. Then, another 2 or 3 sutures, now including the esophagus, completing the valve. There was no mortality, convertion or complications inherent to the method. We conclude that videolaparoscopic fundoplication is a safe procedure for the definitive treatment of GERD, and provides the advantages of a simple surgical technique, with a low incidence of complications.
\end{abstract}

Key words: Disease by esophagic reflux; Gastroesophagic reflux; Esophagite by reflux; Inferior esophagic esfincter; Laparoscopy. 


\section{REFERÊNCIAS}

1. Azevedo JRS. A videolaparoscopia mudou a indicação da doença do refluxo gastroesofagiano? Boletim Informativo Colégio Brasileiro de Cirurgiões 1997, Julho/Setembro.

2. Robinson M, Dahba B, Avner DA. Comparison of lanzoprazole and ranitidine in the treatment of erosive oesophagitis. Al Pharm Therap 1995; 9:23-31.

3. Paluzzi MW. Laparoscopic fundoplication at a teaching center: prospective analysis of 103 consecutive patients. Surg Laprosc \& Encosc 1997; 4:363-368.

4. Heading RC. Epidemiology of oesophageal reflux disease. Scand J Gastroent 1989; 24:33-37.

5. Richter JE. Extraoesophageal manifestation of gastroesophageal reflux disease. Clin Perspectives Gastroenterol 1998; 1:28-39.

6. Locke Grrd et al. Prevalence and clinical espectrum of gastroesophageal reflux: a population based study in Olmsted County, Minnesota Gastroenterology 1997; 112:1448-1456

7. Tytgat GNJ. Endoscopy of the esophagus In: Cotton PB et al. ed. Annual of gastroentestinal endoscopy 1990:15-26.

8. Riedel RH. The biopsy diagnosis of gastroesophageal reflux disease, "carditis", and Barrett's esophagus, and sequelae of therapy. Am J Pathol 1996, suplement 1:531551.

9. Vantrappen G, Janssens J. Place de manomètrie dans le diaginostic des troubles moteurs de l'oesophage. Presse Medicale 1989; 12:611-613.

10. Hueder RA, Filipi CJ. Laparoscopic Nissen fundoplication is a effetive treatment for gastroensophageal reflux disease. Ann Surg 1994; 220:472-483.
11. DeMeester TR, Johnson LF, Skyner DB. Patterns of gastroesophageal reflux in healt and disease. Ann Surg 1976; 4:459-470.

12. Miguel PR et al. Esfagomanometria e pHmetria de 24 horas para avaliar a fundoplicatura Lind laparoscópica na doença do refluxo gastroesofágico. Rev Col Bras Cir 1998;25:241-245.

13. Jamiesson JR, Stein HS, Demeester TR et al. Ambulatory 24 hour esophageal $\mathrm{pH}$ monitoring: normal values, optimal thresholds, specificity and reproductibility. Am J Gastroenterol 1992; 87:1102-1111.

14. Mc Kernan JB, Champion JK. Laparoscopic antireflux surgery Amer Surg 1995; 5:530-536.

15. Donahus PE, Samelson S, Nyhus LM, Bombeck T. The floppy Nissen fundoplication. Arch Surg 1985;120:663-668.

Endereço para correspondência

Dr. Hamilton Luiz Xavier Funes

Rua Cândido Carneiro, 663 - Bom Jesus

15014-200 - São José do Rio Preto - SP 